\title{
The composition and metabolic phenotype of Neisseria gonorrhoeae biofilms
}

\section{Megan L. Falsetta ${ }^{1+}$, ChristopherT. Steichen ${ }^{1+}$, Alastair G. McEwan ${ }^{2}$, Christine Cho ${ }^{1}$, Margaret Ketterer ${ }^{1}$, Jianqiang Shao ${ }^{1}$, Jason Hunt ${ }^{1}$, Michael P. Jennings ${ }^{3}$ and Michael A. Apicella ${ }^{1 *}$}

\author{
1 Department of Microbiology, The University of lowa, lowa City, IA, USA \\ 2 School of Chemistry and Molecular Biosciences, The University of Queensland, Brisbane, QLD, Australia \\ 3 Institute for Glycomics, Griffith University, Gold Coast, QLD, Australia
}

\section{Edited by:}

Cynthia N. Cornelissen, Virginia Commonwealth University School of Medicine, USA

\section{Reviewed by:}

Robert A. Nicholas, University of North Carolina at Chapel Hill, USA

Virginia Clark, University of Rochester, USA

\section{${ }^{*}$ Correspondence:}

Michael A. Apicella, Department of Microbiology, The University of lowa 51 Newton Road, BSB 3-403, lowa City, IA 52242, USA

e-mail:michael-apicella@uiowa.edu ${ }^{\dagger}$ Megan L. Falsetta and Christopher T. Steichen have contributed equally to this work
Neisseria gonorrhoeae has been shown to form biofilms during cervical infection. Thus, biofilm formation may play an important role in the infection of women. The ability of N. gonorrhoeae to form membrane blebs is crucial to biofilm formation. Blebs contain DNA and outer membrane structures, which have been shown to be major constituents of the biofilm matrix. The organism expresses a DNA thermonuclease that is involved in remodeling of the biofilm matrix. Comparison of the transcriptional profiles of gonococcal biofilms and planktonic runoff indicate that genes involved in anaerobic metabolism and oxidative stress tolerance are more highly expressed in biofilm. The expression of aniA, $c c p$, and norB, which encode nitrite reductase, cytochrome $c$ peroxidase, and nitric oxide reductase respectively, is required for mature biofilm formation over glass and human cervical cells. In addition, anaerobic respiration occurs in the substratum of gonococcal biofilms and disruption of the nor B gene required for anaerobic respiration, results in a severe biofilm attenuation phenotype. It has been demonstrated that accumulation of nitric oxide (NO) contributes to the phenotype of a norB mutant and can retard biofilm formation. However, $\mathrm{NO}$ can also enhance biofilm formation, and this is largely dependent on the concentration and donation rate or steady-state kinetics of NO. The majority of the genes involved in gonococcal oxidative stress tolerance are also required for normal biofilm formation, as mutations in the following genes result in attenuated biofilm formation over cervical cells and/or glass: oxyR, gor, prx, $m n t A B C, \operatorname{tr} x B$, and estD. Overall, biofilm formation appears to be an adaptation for coping with the environmental stresses present in the female genitourinary tract. Therefore, this review will discuss the studies, which describe the composition and metabolic phenotype of gonococcal biofilms.

Keywords: Neisseria gonorrhoeae, biofilm, DNA, thermonuclease, matrix, anaerobic, enzymes

\section{INTRODUCTION}

Gonococcal infection has been recognized by different human societies as a distinct disease for over 4,000 years (Handsfield and Sparling, 2005). Gonorrhea, the infection caused by the Neisseria gonorrhoeae, is the second most commonly reported notifiable disease in the United States today with 355,991 cases of gonorrhea reported in 2007 (Anonymous, 2008). The infection rate in the United States has not changed since 1994 (Anonymous, 2008). Gonococcal disease in young women (age 15-25) has many consequences including increasing the risk for infertility, ectopic pregnancy, tubo-ovarian abscesses, and HIV infection (Holmes et al., 1999). In some cities in the US, infection rates in this age group approaches and exceeds $1.5 \%$ of the population (Jennings et al., 2010). Annually worldwide, approximately 69 million new cases of gonorrhea occur with the greatest number in Southeast Asia and Sub-Saharan Africa (Gerbase et al., 1998). Infection in $99 \%$ of men is symptomatic and treatment is sought quickly (Holmes et al., 1999). In contrast, up to $40 \%$ of infected women frequently exhibit no noticeable symptoms and are susceptible to chronic complications from undiagnosed gonorrhea (Holmes et al., 1999; Anonymous, 2008). The reservoir of asymptomatic infected individuals and antimicrobial resistance are two of the major contributors to the spread of gonorrhea (Holmes et al., 1999).

Our laboratory has shown that cervical gonorrhea involves a biofilm component (Greiner et al., 2005; Steichen et al., 2008). This contributes to persistence, and it has been established in the literature that biofilms are inherently resistant to antimicrobials (Ceri et al., 1999; Schierholz et al., 1999; Dunne, 2002), although this has not been directly tested for Neisseria. We now have evidence that the gonococcal biofilm matrix is composed of shed N. gonorrhoeae outer membrane and DNA, which can be remodeled by a chromosomally encoded nuclease. In addition, nitric oxide (NO) appears to be a factor that stimulates biofilm dispersal (Falsetta et al., 2010). The consequences of gonorrhea are significant, negatively affecting the reproductive health of infected individuals and helping to increase the spread of other sexually transmitted diseases, such as HIV1 (Holmes et al., 1999). Since this organism is an obligate human pathogen, the potential to eliminate gonorrhea with an effective vaccine is theoretically possible. However, through its close association with the human, the organism has evolved a repertoire of mechanisms to evade the human immune system including 
antigenic and phase variation, molecular mimicry, resistance to host oxidative processes and the ability to incorporate DNA from its environment all of which make effective vaccine development problematic and unlikely to occur in the near future (Handsfield and Sparling, 2005). Two features of this infection make eradication very difficult: asymptomatic carriage and an increasing number of antimicrobial resistance strains (Holmes et al., 1999). Every decade since the 1950s, the CDC recommendations for the treatment of gonorrhea have become more aggressive and more expensive, from low dose penicillin $\mathrm{G}$ in the 1950s to comparatively expensive, injectable, long acting cephalosporins today (Anonymous, 2008). Resistance to these cephalosporins is now being reported (Bala and Sood, 2010; Golparian et al., 2010). Novel approaches to therapy are needed, and it is possible that greater understanding of the critical points in the pathogenesis of human infection, as well as improving the methods of diagnosis in asymptomatic women, may enable better multi-drug therapies to be applied. New multi-drug therapies would ideally reduce the emergence of resistance and eliminate the asymptomatic (female) carrier.

Since its discovery in 1879 until the 1990s, N. gonorrhoeae was considered an extracellular pathogen. Our work, and that of others, has shown that this organism can infect human genital cells in men and women and that these processes are crucial in pathogenesis (Edwards and Apicella, 2004). We have recently shown that the organism has the ability to form biofilms on the cervical epithelial cell surface in women (Steichen et al., 2008). It is well known that organisms within biofilms can be significantly more resistant to antimicrobials than planktonic organisms (Tart and Wozniak, 2008). As we will discuss in this review, the gonococcal biofilm matrix is composed of shed outer membranes and a matrix composed of DNA released from these blebs (Steichen et al., 2008). During the course of our work, we have identified a nuclease encoded in the gonococcal chromosome, which appears to be involved in remodeling of the biofilm matrix. We believe, but have not proven, that the activity of this nuclease is regulated. Complementary studies performed in our laboratories have examined the metabolic phenotypes of gonococcal biofilms, subsequently identifying a number of enzymes crucial for biofilm development (Seib et al., 2004, 2005, 2006, 2007; Wu et al., 2005, 2006, 2010; Lim et al., 2008; Potter et al., 2009a,b; Falsetta et al., 2010). In addition, our laboratories have shown that nitric oxide plays a dual role in biofilm formation. In the predominately anaerobic environment of the human cervix, nitric oxide (NO) plays a role as an electron acceptor in a respiratory pathway necessary for the survival of the gonococcus. Our studies have shown that nitric oxide reductase (NorB) is critical for production of a biofilm (Falsetta et al., 2009, 2010). NorB is involved in the reduction of nitric oxide (NO) to nitrous oxide $\left(\mathrm{N}_{2} \mathrm{O}\right)$ and mutants in norB accumulate $\mathrm{NO}$ resulting in biofilm dispersal (Householder et al., 2000; Falsetta et al., 2009, 2010), which can be reversed by an NO scavenger. Our studies have also shown that NO is involved in biofilm dispersal (Falsetta et al., 2009, 2010). Moreover, transcriptomic and proteomic analyses of N. gonorrhoeae biofilm formation using in vitro models have shown a dynamic reorganization of gene and protein expression profiles between planktonic and biofilm populations. These data have provided us with new insights into the underlying biology of biofilm formation and have identified sets of proteins that are up- and/or down-regulated in these two states. For example, proteins such as nitrite reductase (AniA) and the RpiR transcriptional regulator are found to significantly increase in biofilms, while ferric enterobactin receptor (FetA) and transferrin-binding protein $\mathrm{A}(\mathrm{TbpA})$ appear more highly expressed in planktonic organisms. This review will focus on the available studies of N. gonorrhoeae biofilm, which have been conducted almost exclusively by our laboratories. Here we describe the unique structure, composition, and metabolic phenotype of gonococcal biofilms, which contributes to colonization and persistence in women, and may be paramount to its success as a pathogen in the cervical environment.

\section{THE STRUCTURE AND COMPOSITION OF GONOCOCCAL BIOFILMS}

We have investigated the matrix components of the N. gonorrhoeae biofilm. The organism lacks genes capable of producing exopolysaccharides. Instead, our studies have shown that membrane blebbing is necessary for mature biofilm formation (Steichen et al., 2008). Previously, Dorward et al. (1989) showed that N. gonorrhoeae membrane blebs were associated with large amounts of DNA. This prompted us to examine the gonococcal biofilm for the presence of DNA. DNA has been shown to be a major constituent of Pseudomonas aeruginosa and non-typeable Haemophilus influenzae biofilms. Our experiments showed bovine pancreatic DNaseI can rapidly disrupt established N. gonorrhoeae biofilms under continuous-flow conditions (Figure 1). In addition, confocal microscopy and electron microscopic studies demonstrated that DNA is a major component of the N. gonorrhoeae biofilm (Figure 2). Concomitant with this, we discovered that a hypothetical protein (NGO0969) encoded in N. gonorrhoeae 1291 genome had $25 \%$ identity and $40 \%$ similarity to the Staphylococcus aureus secreted thermonuclease that we have denoted Nuc (Steichen et al., 2011). The nuc gene appears to be co-transcribed in an operon containing at least six other genes, NGO0968-NGO0974. In order to determine if the protein encoded by the NGO0969 open reading frame (ORF) is in fact able to degrade DNA, we expressed this protein, designated Nuc, in Escherichia coli. The ORF was amplified starting from codon \#36 as the online signal sequence predictor, SignalP 3.0, predicts the first 34 amino acids to be a TAT dependent signal peptide. Our results using the purified Nuc demonstrated it is ability to digest a single stranded DNA oligonucleotide as well as chromosomal DNA from a variety of prokaryotic and eukaryotic sources. To determine if activity of the Nuc protein requires the presence of divalent cations, identical experiments were run with EDTA (added as a chelating agent) to a final concentration of $4 \mathrm{mM}$. The addition of EDTA completely inhibited the activity of Nuc and no increase in fluorescence was observed. This indicates that the divalent cations $\left(\mathrm{Ca}^{2+}\right.$ and/or $\mathrm{Mg}^{2+}$ ) supplied in the buffer are necessary for Nuc DNA digestion activity. No nuclease activity was detected enzymatically in planktonic runoff or broth supernates, yet activity was present in N. gonorrhoeae lysates. Our biochemical studies also indicate that the methylation state of the DNA in the gonococcal biofilm is a factor in determining the susceptibility of gonococcal DNA to digestion (Steichen et al., 2011). The gonococcus is known to actively secrete DNA (Hamilton et al., 2005). Given that Nuc has a $\mathrm{N}$-terminal tat dependent signal peptide, we postulate that as 

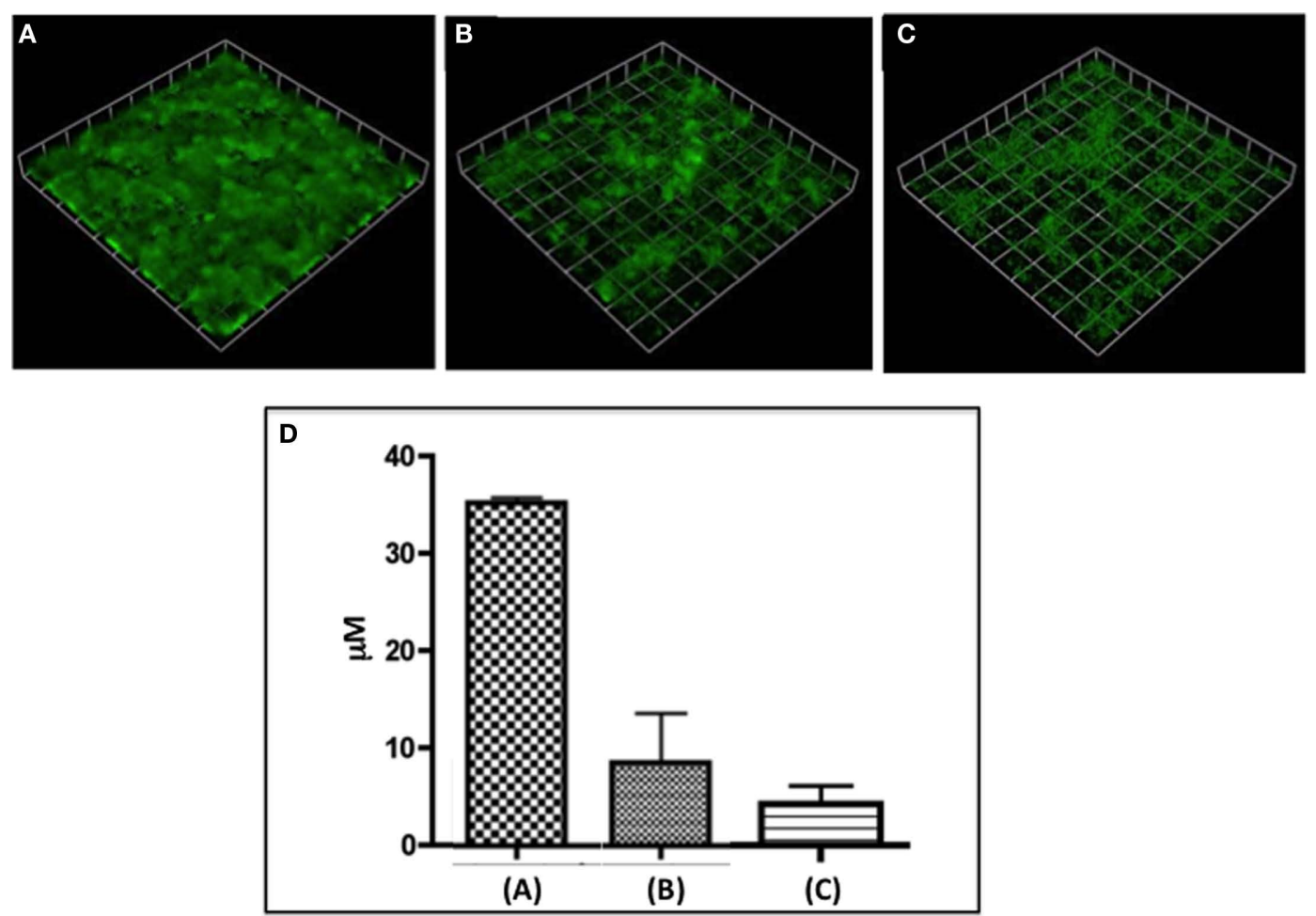

FIGURE 1 | It shows $N$. gonorrhoeae 1291 biofilms either untreated (A) or treated for $5 \mathrm{~h}$ with $2 \mathrm{U} / \mathrm{ml} \mathrm{DNasel} \mathrm{(B)} \mathrm{or} 5 \mu \mathrm{gm} / \mathrm{ml}$ Neisseria nuclease (C)

(D) Shows a Comstat analysis of the average height of the respective biofilms. This figure demonstrates significant loss of biofilm after treatment with either nuclease.
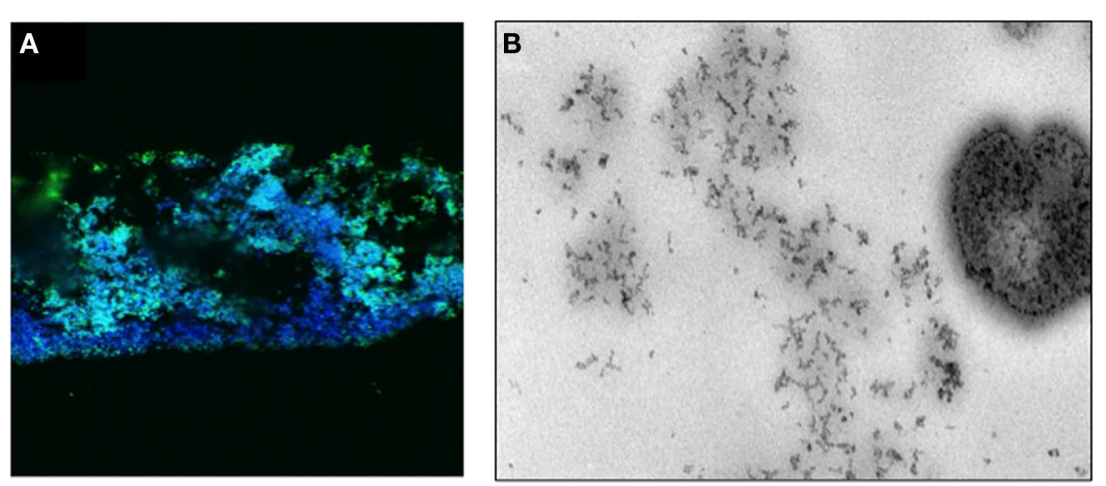

FIGURE 2 | (A) shows a confocal micrograph of a frozen section of a biofilm of N. gonorrhoeae 1291 expressing pGFP (green) and stained with DAPI (blue). (B) Shows an electron micrograph of a cryosection of a 48-h N. gonorrhoeae 1291 biofilm stained propidium iodide. The section was treated with RNase and proteinase K sequentially prior to staining with propidium iodide. The speckled DNA staining can be seen in the matrix substance in the biofilm and in the bacteria seen in the image.

DNA is secreted by the gonococcus, it is modified by the nuclease in the periplasm prior to secretion. The degree of remodeling determines the amount of DNA incorporated into the biofilm. We have evidence that this is a regulated process, as we have found that the abundance of DNA varies depending on the age and developmental state of the biofilm. Using DAPI staining, we demonstrated that $48 \mathrm{~h}$ N. gonorrhoeae 1291 biofilms contain DNA in the matrix, and that the amount of DNA is elevated in the 1291 nuc mutant (Figure 2). In separate experiments, quantitative analysis of a collected $z$-series was performed using COMSTAT software, to obtain numeric values for the total biomass and average thickness of these biofilms. Statistical analysis of these $z$-series using Student's $t$-test indicated that the nuc mutant produces a biofilm with greater biomass and average thickness than that of the parent strain $p=0.01$. Chromosomal complementation of the $n u c$ mutant with nuc results in biofilms with height and mass similar to the wild type strain and significantly different than the $n u c$ mutant $(p<0.001)$. 


\section{THE METABOLIC PHENOTYPE OF GONOCOCCAL BIOFILMS}

Prior to our work, little was understood about the mechanisms that contribute to or govern biofilm formation in N. gonorrhoeae. Much of the current biofilm literature suggests that biofilms exhibit unique patterns of gene expression that dictate community behaviors, including resistance to antimicrobials and host immune defenses (Whiteley et al., 1999; Beloin and Ghigo, 2005; An and Parsek, 2007). Using microarray analysis, 83 genes were identified that met our criteria for differential expression (fold-change $\geq 2.0$ and $p \leq 0.05$ ) when biofilms grown over glass were compared to planktonic cells filtered from the biofilm effluent, the vast majority of these genes were hypothetical $(57.8 \%)$, although many of the proteins encoded by these genes could play important roles in biofilm formation (Falsetta et al., 2009). We found that genes involved in anaerobic respiration ( $a n i A, c c p$, and nor $B$ ) were highly up-regulated during biofilm formation, while genes involved in aerobic respiration were down-regulated (nuo operon; Falsetta et al., 2009). qRT-PCR was used to confirm expression of these genes over glass and to examine expression in a more relevant model system (growth over transformed human cervical epithelial cell, THCEC). We found that the pattern of regulation was similar over glass and over THCEC (Falsetta et al., 2009). This finding would suggest that expression of these genes is relevant to infection. These data also indicate that our continuous-flow system for biofilm growth over glass may be a suitable model for biofilm formation when the experimental design does not permit the use of cervical cells. For example, this system may be a useful tool for modeling biofilm behaviors during treatment with compounds that are toxic to or impair the growth of primary cervical cells.

The pathogenic Neisseria have evolved a number of mechanisms to deal with oxidative stress (Figure 3 ). High levels of expression of aniA and norB during growth as a biofilm indicates that anaerobic respiration occurs in gonococcal biofilms (Householder et al., 1999, 2000). N. gonorrhoeae is often isolated in the presence of obligate anaerobes and the cervical environment is presumed to be oxygen limited (Smith, 1975). Thus, anaerobic growth is important for gonococcal survival in vivo. Anaerobic respiration also contributes to the ability of the gonococcus to tolerate oxidative stress, as NorB can rapidly achieve a NO steady-state that reduces proinflammatory concentrations of $\mathrm{NO}$ to concentrations that are not inflammatory (Cardinale and Clark, 2005). Like NorB, Ccp also reduces an oxidant present in the cervical environment. Ccp reduces $\mathrm{H}_{2} \mathrm{O}_{2}$, which is produced by host PMNs and the Lactobacillus species that also inhabit the female genitourinary tract (Carreras et al., 1994b). Ccp expression only occurs under anaerobic conditions (Turner et al., 2003), and this would be consistent with the use of hydrogen peroxide as an electron acceptor when oxygen is limited.

The role of Ccp in the defense against hydrogen peroxide is redundant, as gonococcal catalase can also reduce $\mathrm{H}_{2} \mathrm{O}_{2}$ (Seib et al., 2006). If mutations are made in either the $c c p$ or the katA (catalase) gene, the resulting strains become sensitive to $\mathrm{H}_{2} \mathrm{O}_{2}$-mediated killing (Seib et al., 2006). Moreover, a ccp/katA double mutant is more sensitive to $\mathrm{H}_{2} \mathrm{O}_{2}$ than either single mutant (Seib et al., 2006). This may help to account for the observation that the ccp::kan mutant is only partially impaired in its ability to form biofilms over glass. Thus, we would expect that a $c c p / k a t A$ mutant may be further impaired in its ability to form biofilm, although this has not

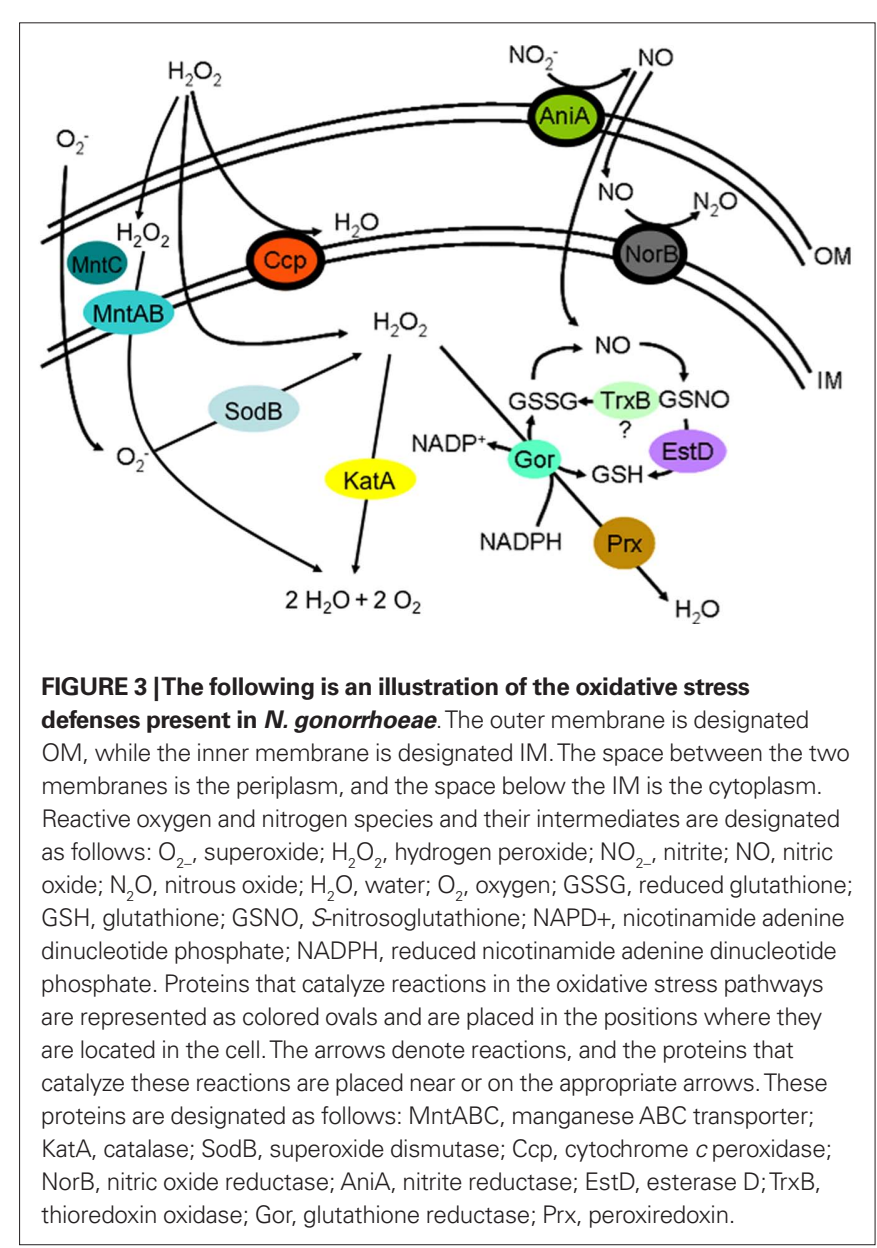

been directly tested. The role of AniA is not functionally redundant and yet the biofilm phenotype of the aniA::kan mutant is indistinguishable from the phenotype of the $c c p:$ :kan mutant. Although a partially attenuated phenotype in the $c c p:$ :kan mutant may be simply explained by functional redundancy, this does not account for a similar phenotype in the aniA::kan mutant. Other organisms also catalyze anaerobic respiration during growth as a biofilm (Hassett et al., 2002; Filiatrault et al., 2006) and our observations would be consistent with a significant role for energy generation via nitrite in the gonococcal biofilm. It has become increasingly evident that anaerobic respiration is critical for biofilm formation in a number of organisms, including the biofilm paradigm organism, P. aeruginosa (Hassett et al., 2002; Yoon et al., 2002; Filiatrault et al., 2006; Van Alst et al., 2007). Evidence suggests that anaerobic respiration is the primary mode of growth for $P$. aeruginosa during cystic fibrosis infection. This mode of growth may contribute to the antimicrobial resistance of $P$. aeruginosa biofilms, as cells that undergo anaerobic respiration are concentrated near the biofilm substratum, are less metabolically active, and are likely less susceptible to antimicrobials that target active cellular processes (Werner et al., 2004; Rani et al., 2007). Oxygen only penetrates approximately the first $50 \mu \mathrm{M}$ of $P$. aeruginosa biofilms (Werner et al., 2004), although these biofilms typically achieve thicknesses of approximately $100 \mu \mathrm{M}$ or more (Davies et al., 1998; Sauer et al., 2002). This observation may help to explain why the aniA::kan 
mutant forms biofilms with biomasses that are similar to wild type, yet these biofilms are significantly thinner than the wild type. If oxygen cannot penetrate the entire depth of the biofilm, the thicknesses of the biofilm may be limited by the availability of oxygen in mutants that cannot respire anaerobically. The thickness of an aniA::kan mutant biofilm does exceed $60 \mu \mathrm{M}$, which appears to correlate with the findings in P. aeruginosa (Werner et al., 2004).

Biofilm formation was most severely attenuated in the norB::kan mutant, as this mutant had significantly less biomass and lower average thicknesses than the wild type (Falsetta et al., 2009). This was initially puzzling, as norB and aniA encode components of the partial denitrification pathway found in pathogenic Neisseria and previous studies demonstrated that mutations in either norB or aniA resulted in strains that were unable to respire anaerobically (Householder et al., 1999, 2000). However, there was one obvious difference in these two mutant strains. AniA reduces nitrite to NO (Mellies et al., 1997), which is often toxic to many bacterial species (Fang, 1997; MacMicking et al., 1997; Zumft, 1997; Davies et al., 1998). NorB then reduces NO to nitrous oxide (Householder et al., 2000), which is not generally considered to be toxic (Seib et al., 2006). A mutation in norB would render the gonococcus unable to reduce AniA-generated NO. Thus, we considered the possibility that the accumulation of NO may be toxic or could impair biofilm formation in the norB::kan mutant through another mechanism, especially considering that $\mathrm{NO}$ is an important signaling molecule in eukaryotes (MacMicking et al., 1997; Davis et al., 2001). $N$. gonorrhoeae is presumed to be inherently resistant to NO, and a norB::kan mutant survives incubation under anaerobic conditions, which should result in the accumulation of AniA-generated NO
(Householder et al., 2000). Thus, it would be unlikely that the accumulation of NO simply impairs biofilm formation through cytotoxicity. Furthermore, the gonococcus produces a variety of proteins that are involved in resistance to nitrosative stress, including esterase $\mathrm{D}$, which is regulated by $\mathrm{NmlR}$ and is involved in the tolerance of nitrite and $S$-nitrosogluthathione, as estD::kan mutant is sensitive to both (Potter et al., 2009a).

We also used a scanning electron microscopy (SEM, Figure 4) technique developed by our laboratory to evaluate the structure of the mutant biofilms (Srikhanta et al., 2009). We found that the architecture of the mutant biofilms was distinctly different from that of wild type biofilms (Figure 4). Specifically, wild type biofilms were confluent over the glass surface of attachment and the ability to visualize individual biofilm cells was obscured by copious amounts membrane blebs, which are a large constituent of the biofilm matrix. The $c c p:: k a n$ and aniA::kan mutants were both less confluent and contained fewer membrane blebs than the wild type, and the majority of the biofilm cells were exposed and not enmeshed within the biofilm matrix. The norB::kan mutant was much more severely attenuated than the aniA::kan or ccp::kan mutant. Few norB::kan cells were adherent to the surface of attachment and almost no matrix material was associated with the cells, although there did appear to be blebs that were associated with the glass surface. Cells in the norB::kan mutant biofilms also exhibited an unusual morphology, where these cells appeared to be slightly elongated and smaller in size than the cells in the aniA::kan and ccp::kan biofilms. The accumulation of NO may also explain the striking morphology of the norB::kan mutant biofilms. These findings again confirm that the norB::kan mutant is more severely attenuated than either the
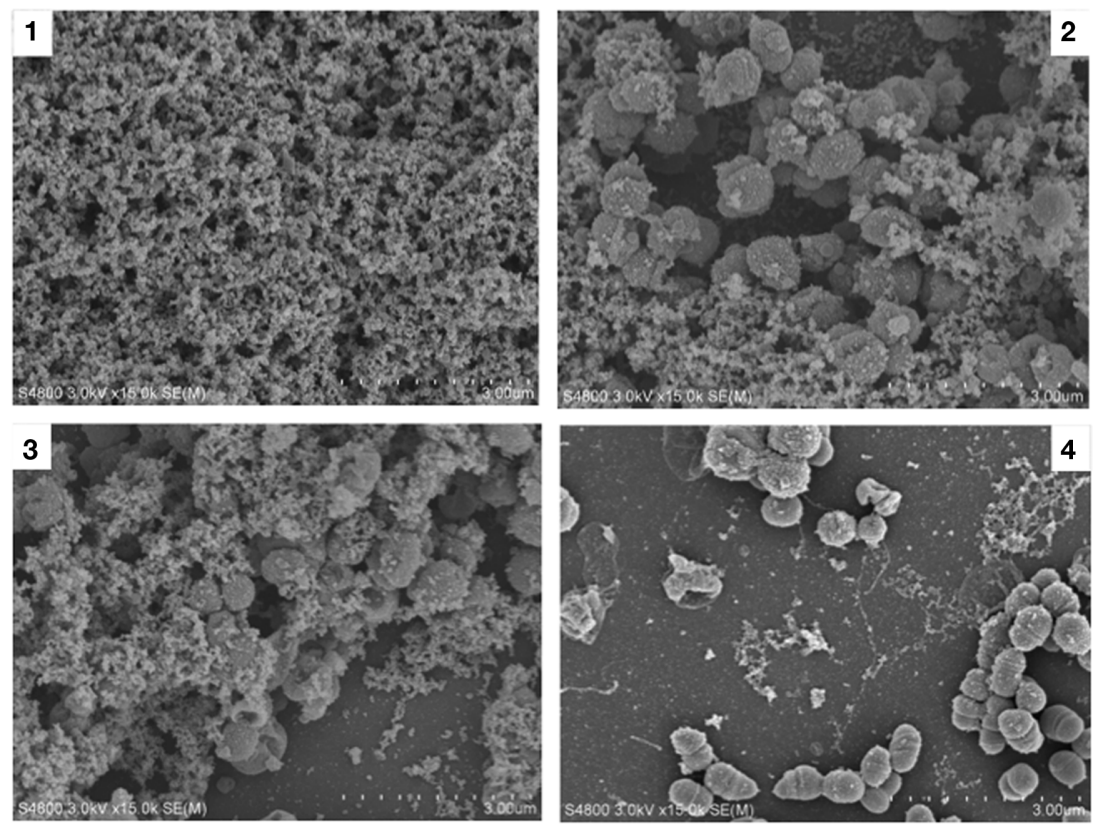

FIGURE 4 | Scanning electron microscopic examinations of biofilms produced by the wild type, aniA, $\boldsymbol{c c p}$, and norB mutant. Panel 1 is a scanning electron micrograph of a N. gonorrhoeae 1291 48-h wild type biofilm taken at 15,000× magnification. Panel 2 depicts the aniA::kan mutant, while panel 3 depicts the ccp::kan mutant, and panel 4 depicts the norB::kan mutant. The morphology of all three mutants is distinctly different that the wild type, however it is apparent that norB::kan mutant is more severely attenuated than the aniA::kan and ccp::kan mutant. 


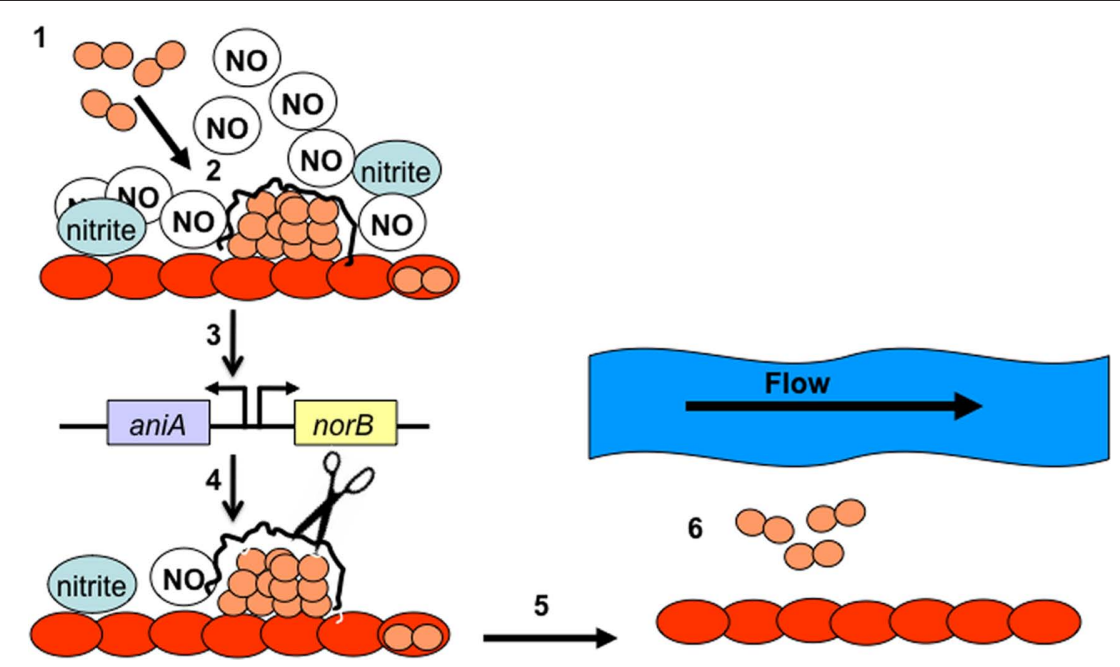

FIGURE 5 | It shows our theoretical model of the roles of AniA, NorB, and NO in remodeling of biofilm structure. During step 1, N. gonorrhoeae (depicted in pink) binds to the surface of the cervical cells (depicted in red). During step 2, these cells differentiate into a biofilm, producing a biofilm matrix. In step 3 biofilm formation turns on transcription of ani $A$ and nor $B$, which produces and modulates the concentration of NO in the surrounding media. In step 4, low levels of NO signal dispersal of the biofilm, which likely occurs through activation of the gonococcal nuclease and degradation of the biofilm DNA matrix (step 5). In step 6, cells that are released from the biofilm may be swept away in bodily secretions, allowing these cells to potentially colonize new areas of cervical tissue.
aniA::kan or $c c p:$ :kan mutant when grown under continuous-flow conditions in a biofilm. To also test biofilm formation in a more relevant system, we infected THCEC with our mutant and wild type strains. We found that all three mutants had significantly reduced biomasses and average thicknesses compared to the wild type when grown over THCEC for $48 \mathrm{~h}$ (Falsetta et al., 2009). This suggests that the role of these genes is more critical in an infection model, which better resembles the conditions present in the host environment.

The striking phenotype of the norB::kan mutant led us to investigate the role of NO in N. gonorrhoeae biofilms. We hypothesized that NO affected biofilm formation through another mechanism. The $P$. aeruginosa biofilm literature suggested that sublethal concentrations of NO may prevent biofilm formation or facilitate biofilm dispersal (Barraud et al., 2006). Therefore, we attempted to rescue biofilm formation in the norB::kan mutant by treating these biofilms with an NO quencher. We found that treatment with PTIO, an NO scavenger, improved biofilm formation in this mutant (Falsetta et al., 2009). Although PTIO improved biofilm formation, biofilms were not completely restored to wild type levels in the presence of PTIO (Falsetta et al., 2009). Wild type biofilms were also treated with the NO donor, sodium nitroprusside (SNP), at the beginning of biofilm formation and after $24 \mathrm{~h}$ of biofilm formation to determine if $\mathrm{NO}$ could also impair biofilm formation in the wild type. SNP was initially selected, because analogous biofilm studies performed with $P$. aeruginosa demonstrated that SNP was an effective NO donor that elicited biofilm dispersal. We found that treatment at the start of biofilm impaired biofilm formation, but did not completely inhibit biofilm formation. Treatment with SNP after $24 \mathrm{~h}$ of biofilm formation resulted in biofilms with less biomass and lower average thicknesses than untreated biofilms (Falsetta et al., 2009). This indicates that the introduction of $\mathrm{NO}$ after $24 \mathrm{~h}$ of biofilm either halts biofilm development or causes the biofilm to disperse. We also considered the possibility that NO could inhibit cytochrome oxidase, thus impairing aerobic respiration in the norB::kan mutant. However, we found that there was no defect in the growth of the norB::kan mutant when it was cultured under oxygen tension conditions that were similar to those present in our biofilm system (Falsetta et al., 2009). Treatment with $20 \mu \mathrm{M}$ concentrations and higher (up to $1 \mathrm{mM}$ ) of SNP completely prevented biofilm formation, if it was administered at the start of the biofilm (Falsetta et al., 2009). These findings indicate that higher concentrations of NO could completely block biofilm formation in N. gonorrhoeae. $P$. aeruginosa, which is inherently more sensitive to NO, can form better biofilms in the presence of high concentrations of $\mathrm{NO}$ (Barraud et al., 2006). When we considered this, it occurred to us that anaerobic respiration would not be immediately induced in gonococcal biofilms, as aniA and nor $B$ are repressed under aerobic growth conditions (Householder et al., 2000; Barraud et al., 2006). Thus, we constructed a fluorescent transcriptional fusion to the aniA gene ( $a n i A^{\prime}-^{\prime} g f p$ ) to monitor the induction of anaerobic respiration in biofilms (Falsetta et al., 2010). We used light microscopy to monitor induction in overnight plate cultures, the biofilm inoculum, and biofilms grown for 24 and $48 \mathrm{~h}$. We were unable to detect fluorescence in plate cultures or the biofilm inoculum. However, we could readily detect GFP in biofilms grown for 24 and $48 \mathrm{~h}$. These data indicate that N. gonorrhoeae biofilms become anaerobic over time, as aniA expression was induced between 0 and $24 \mathrm{~h}$ of biofilm formation (Falsetta et al., 2010). Gradual induction of anaerobic respiration in biofilms could explain why immediate treatment with low concentrations of NO was partially inhibitory, while high concentrations completely inhibited biofilm formation. However, we observed that low doses of SNP halted biofilm formation in 24-h biofilms that were likely expressing aniA and undergoing anaerobic respiration (Falsetta et al., 2010). This suggests that the effect of NO on 
N. 'gonorrhoeae biofilms is not dictated solely by NO toxicity. This agrees with our data that indicate that the norB::kan mutant is not impaired in its ability to grow under microaerobic conditions. Microaerobic conditions classically been defined as those of low oxygen tension (e.g., between 5 and $27 \mathrm{~mm} \mathrm{Hg}$ ).

We also used the $a n i A^{\prime}{ }^{\prime} g f p$ fusion strain to evaluate the profile of anaerobic metabolism in biofilm. P. aeruginosa biofilms are stratified and use a combination of anaerobic and aerobic metabolism (Rani et al., 2007). Protein synthesis occurs in the uppermost 30-60 $\mu \mathrm{m}$ of biofilm, while anaerobic/metabolically inactive cells comprise the majority of the biofilm and are localized near the substratum (Werner et al., 2004). To determine if this was similar for $N$. gonorrhoeae, we grew the ani $A^{\prime}$ - $^{\prime} g \mathrm{fp}$ fusion strain for $48 \mathrm{~h}$ and then stained the biofilm with 2C3 (H.8 antibody) to visualize all cells in the biofilm. We found that the majority of the biofilm cells expressed aniA, and that cells expressing aniA were located near the surface of attachment in the substratum of biofilm (Falsetta et al., 2010). The uppermost region of the biofilm was almost entirely comprised of cells that did not express aniA (Falsetta et al., 2010). This indicates that cells near the fluid-flow interface primarily use aerobic metabolism. This pattern is similar to the patterns observed in P. aeruginosa biofilms (Werner et al., 2004; Rani et al., 2007) aniA expression also did not localize to areas where there were gaps in the biofilm, indicating the presence of water channels. Transcripts of aniA and nor $B$ are virtually undetectable under aerobic growth conditions (Householder et al., 1999, 2000; Whitehead et al., 2007). Thus, it is no surprise that aniA is not expressed at the biofilm-fluid-flow interface where oxygen would be abundant. The dissolved oxygen concentrations of media entering and exiting the biofilm chamber were calculated and it was found that the concentration of oxygen was well above anaerobic or microaerobic concentrations (Falsetta et al., 2009). Although mutations in anaerobic respiratory genes can cause severe impairment of biofilm formation, especially when cultured in the presence of host cells, these mutations do not completely prevent biofilm formation (Falsetta et al., 2009, 2010). This finding reflects the metabolic profile of $N$. gonorrhoeae biofilms, which catalyze both anaerobic and aerobic respiration. Expression of aniA is not induced in the initial biofilm inoculum, which suggests that biofilms may rely on aerobic respiration early during an infection, prior to the establishment of a biofilm and transcription of the anaerobic respiratory genes.

To further examine the effect of NO on biofilm formation, a method was devised to induce anaerobic respiration in biofilms before evaluating the effect of NO. We grew biofilms for $24 \mathrm{~h}$ in the presence of nitrite, then transitioned these biofilms to media without nitrite, which either was or was not supplemented with $20 \mu \mathrm{M}$ SNP. We previously determined that growing biofilms in the presence of nitrite for $24 \mathrm{~h}$ induces expression of aniA (Falsetta et al., 2009). Biofilm formation was enhanced by the addition of SNP under these conditions (Falsetta et al., 2010). It appears that treatment with high doses of SNP can enhance biofilm formation, if anaerobic respiration has been initiated. Anaerobic respiration likely allows nor $B$ to be transcribed at the levels necessary to effectively reduce NO concentrations. This observation further supports the hypothesis that $N$. gonorrhoeae biofilms are important for and contribute to oxidative stress tolerance during infection.
In our aim to develop a better model for studying the impact of NO on biofilm formation, another NO donor (DETA/NO) was selected for the treatment of biofilms. SNP has a relatively short half-life (on the order of minutes) and generates both NO and cyanide, the later of which could be toxic to biofilm cells. However, we did measure the concentrations of NO donated from our SNP stocks and determined that authentic $\mathrm{NO}$ was generated and that the concentration is not likely to exceed to $20 \mathrm{nM}$ in the biofilm media (Falsetta et al., 2010). SNP was initially selected, so we could perform experiments that would parallel those published for $P$. aeruginosa biofilms, and it can easily be obtained in quantities sufficient to treat biofilm. However, DETA/NO is a more ideal NO donor, as it has a half-life of $20 \mathrm{~h}$ (Cardinale and Clark, 2005) and should donate NO gradually during the initial stages of biofilm development. This would presumably better reflect the conditions present in vivo where consistent (low) concentrations of NO would be supplied by cervical cells and PMNs (Carreras et al., 1994b; Fang, 1997; Seib et al., 2006). Cardinale and Clark (2005) have shown that the gonococcus can achieve and maintain a NO steady-state of approximately $85 \mathrm{nM}$ after addition of $0.3 \mathrm{mM}$ DETA/NO, when the cells are grown on plates. They also demonstrated that the steadystate achieved is independent of $\mathrm{pH}$, but is dependent on the concentration of nitrite, if the initial concentration of nitrite supplied is below $1 \mathrm{mM}$. Due to the substantial differences in experimental design between our study and that of Cardinale and Clark (2005, i.e., growth as biofilm versus growth on plates), it is difficult to draw specific conclusions about the NO steady-state present in our biofilm system. However, we did measure the concentration of NO as it was donated from our DETA/NO stocks, and we determined that the average concentration supplied to the biofilm is between 15 and $22 \mathrm{nM}$. We are not aware of any studies that have directly measured the NO concentrations present in the cervical environment. However, the concentrations of NO supplied by DETA/NO are unlikely to be greater than those present in vivo, and based on the work of Cardinale and Clark (2005) these concentrations would not likely be detrimental to the growth $N$. gonorrhoeae.

We found that $20 \mu \mathrm{M}$ DETA/NO did not prevent biofilm formation, but rather enhanced biofilm formation in the absence of nitrite (Falsetta et al., 2010). This finding suggests that DETA/NO donates $\mathrm{NO}$ at a rate that is easily tolerated during the early stages of biofilm development, before the induction of anaerobic respiration. It is obvious that the concentration of NO, the rate at which it is donated, and the time at which it is administered determines the effect on biofilm formation. Low concentrations of NO prevent biofilm formation, even in the presence of nitrite, while high concentrations enhance biofilm formation if a slow-release NO donor is used or anaerobic respiration is occurring in biofilm (Falsetta et al., 2009, 2010). A model has been proposed that illustrates the potential effects of NO on biofilm formation (Figure 5). This model proposes that NO is abundant when the gonococcus encounters the cervical environment, which elicits biofilm formation, leading to the expression of anaerobic respiratory genes (e.g., aniA and nor $B$ ). Expression of nor $B$ would result in a reduction of the local NO concentration, signaling dispersal of the biofilm, which would likely be mediated by DNA nuclease (Falsetta et al., 2010). However, additional evidence is needed to either support or refute this model, and it is not clear what protein(s) may be sensing the concentration 
of NO in biofilm. We would propose that the NsrR regulator may play a role in sensing the concentration of NO in biofilm, as NsrR is sensitive to $\mathrm{NO}$ (NO-sensitive repressor; Whitehead et al., 2007). When NO is present, NsrR is unable to function as a negative regulator of aniA and nor $B$, which results in the de-repression of aniA and norB expression (Whitehead et al., 2007). It may be possible that NsrR regulates other previously unidentified targets that could play a role in the NO response. Further study is warranted to investigate this hypothesis.

Biofilms treated with DETA/NO from the start of biofilm formation or treated with SNP after $24 \mathrm{~h}$ of growth resembled those grown in the presence of nitrite for $48 \mathrm{~h}$ (Falsetta et al., 2009, 2010). Prior to determining that $N$. gonorrhoeae biofilms catalyze anaerobic respiration, it was observed that nitrite enhanced biofilm formation, although biofilms could form in the absence of nitrite (Greiner et al., 2005). Biofilms grown in the absence of nitrite develop at slower rate than those grown in the presence of nitrite (Greiner et al., 2005). Thus, supplementation of biofilms with nitrite allowed mature biofilms to form by $48 \mathrm{~h}$ post-inoculation. $N$. gonorrhoeae was initially considered to be incapable of anaerobic growth, due to the inability to culture cells under anaerobic conditions (JamesHolmquest et al., 1973). This led to the finding that $N$. gonorrhoeae is unique in that it uses nitrite as a terminal electron acceptor for anaerobic growth, and it is incapable of using nitrate (Knapp and Clark, 1984). However, we found that biofilm growth can be partially restored in an aniA::kan mutant by adding NO to the biofilm media (Falsetta et al., 2009, 2010). This mutant cannot reduce nitrite and similar mutants were considered incapable of anaerobic growth. Previous mutants survived, but did not grow under anaerobic conditions (Householder et al., 1999). Our results suggest that this mutant may be able to grow under anaerobic conditions using NO. NO was not used to supplement the culture media in earlier studies that concluded that an aniA insertion mutant is unable to respire anaerobically (Householder et al., 1999). Although oxygen is abundant in the media of our biofilm system, the majority of the cells in these biofilms use anaerobic respiration (Falsetta et al., $2009,2010)$. Anaerobic respiration occurs in more than two-thirds of the total thickness of $N$. gonorrhoeae biofilms, as visualized by aniA expression (Falsetta et al., 2010). Thus, the partial restoration of biofilm formation in the aniA::kan mutant strongly suggests that these biofilms are able to undergo anaerobic respiration, as the thickness of these biofilm exceeds the thickness of the aerobic portion of gonococcal biofilms. However, supplementation with NO does not fully restore biofilm formation in the aniA::kan mutant (Falsetta et al., 2010). This indicates that we may not have supplemented our media with the optimal concentration of $\mathrm{NO}$, or that nitrite may be the preferred substrate for anaerobic metabolism. The ability to use both nitrite and NO to support anaerobic growth would be of advantage to gonococcal biofilms. If this were the case, NO could be used to support anaerobic growth if the function of AniA was impaired. In support of this hypothesis, some $N$. meningitidis strains possess a frameshift mutation in aniA (Pitcher and Watmough, 2004; Potter et al., 2009a), but are still able to respire anaerobically (Rock et al., 2005, 2007; Deeudom et al., 2006). N. gonorrhoeae biofilms are metabolically heterogeneous and may be able to use a variety of substrates to catalyze anaerobic and aerobic respiration. Biofilm heterogeneity confers advantages for biofilm survival, including the ability of metabolically inactive cells to resist antimicrobial treatment and the host immune response (Werner et al., 2004; Barraud et al., 2006).

If oxygen is abundant in our biofilm system, why do N. gonorrhoeae biofilms predominantly use anaerobic metabolism? The $\mathrm{cbb}_{3}$ type family of cytochrome oxidases has a high affinity for oxygen (Pitcher and Watmough, 2004) and it has been speculated that the oxygen concentration in vivo would have to be considerably lower than the predicted concentration in order to hinder aerobic growth of the gonococcus. Studies that examined the oxygen profiles of $P$. aeruginosa biofilms determined that oxygen is limited in its ability to diffuse into the biofilm (Werner et al., 2004; Barraud et al., 2006). This may be the simplest explanation for the metabolic profile of $N$. gonorrhoeae biofilms, which catalyze aerobic respiration at the fluid-flow interface and anaerobic respiration in the depths of the biofilm. However, the matrix of N. gonorrhoeae biofilm is dramatically different than $P$. aeruginosa biofilm, and it is not clear as to whether the diffusion of oxygen is limited in gonococcal biofilms (Costerton et al., 1999; Steichen et al., 2008). Our results suggest that diffusion of oxygen into N. gonorrhoeae biofilms is limited. This may be a product of slowed diffusion due to the presence of the biofilm matrix and/or aerobic respiration at the biofilm-fluidflow interface, which may consume the oxygen available in the bulk fluid, subsequently impeding diffusion into the biofilm.

Another possibility is that there is a complex interaction between the partial denitrification pathway and aerobic respiration. Moir and co-workers (Rock et al., 2005) have shown that in N. meningitidis that accumulation of $\mathrm{NO}$ above 100-200 nM results in inhibition of oxygen respiration. Thus, it is possible that respiration with nitrite and $\mathrm{NO}$ is of central importance within the biofilm even when oxygen is available and the balance between partial denitrification and aerobic respiration may depend upon the local concentrations of NO in the biofilm. It is interesting to note that the CcoP subunit of the $N$. gonorrhoeae cytochrome $\mathrm{cbb}_{3}$ is a triheme that contains an additional $c$-type cytochrome compared to homologs in other bacteria. The additional heme center seems to be important for electron transfer to the nitrite reductase but not the cytochrome $\mathrm{cbb}_{3}$ (Hopper et al., 2009). The respiratory chain may be organized in this way to allow electron transfer to oxygen or nitrite down a common respiratory pathway that bifurcates just before the terminal reductases.

The induction of anaerobic genes in biofilm may play an important role in oxidative stress tolerance. NorB functions dually in anaerobic respiration and oxidative stress tolerance by reducing NO (Mellies et al., 1997), while Ccp contributes to oxidative stress tolerance by reducing $\mathrm{H}_{2} \mathrm{O}_{2}$ (Turner et al., 2003). Induction of these genes in biofilm suggests that biofilm formation may enhance oxidative stress tolerance in N. gonorrhoeae. Although we did not directly challenge gonococcal biofilms with oxidative stressors (other than NO), we determined that a variety of oxidative stress tolerance genes are required for biofilm formation including $\operatorname{tr} x B$, estD, mntABC, oxyR, prx, and gor (Seib et al., 2004, 2005, 2006, 2007; Wu et al., 2005, 2006, 2010; Lim et al., 2008; Potter et al., 2009a,b; Srikhanta et al., 2009). A reasonable future objective would be to assess the ability of biofilm to withstand oxidative stress as compared to planktonic cells. Ideal oxidants to test would be NO and $\mathrm{H}_{2} \mathrm{O}_{2}$. Despite the varied function of the proteins encoded by these 
genes, all are required for normal biofilm formation. Inhibiting the function of the periplasmic binding protein (MntC) or intermembrane domain (MntAB) of the MntABC transporter severely attenuates biofilm formation over glass. Mutations in either portion of the transporter should impair the ability of the gonococcus to take up Mn. These findings indicate that the antioxidant properties of $\mathrm{Mn}$ help to protect gonococcal biofilms from oxidative stress. However, the mntABC mutants are not as severely impaired as other oxidative stress tolerance mutants, which includes members of the OxyR regulon. This may reflect the relative importance of different oxidative stress tolerance mechanisms in $N$. gonorrhoeae. The OxyR regulator and members of its operon (prx and gor) are also required for robust biofilm formation. oxyR::kan mutant biofilms are indistinguishable from prx::kan or gor::kan mutant biofilms, which suggests that disruption of any member of this operon is sufficient to hinder biofilm formation. The effects of the gor and prx mutations do not appear to be cumulative, although they are not functionally redundant (Seib et al., 2006). However, they do play complementary roles in the reduction of $\mathrm{H}_{2} \mathrm{O}_{2}$ (Seib et al., 2006). Although the gonococcus has several mechanisms for coping with $\mathrm{H}_{2} \mathrm{O}_{2}$ stress, disrupting a single gene involved in $\mathrm{H}_{2} \mathrm{O}_{2}$ tolerance ( $g$ or, prx, or $c c p$ ) can impair biofilm formation (Seib et al., 2005). This suggests that protection against $\mathrm{H}_{2} \mathrm{O}_{2}$ is paramount in biofilms, which may correspond to the prevalence of $\mathrm{H}_{2} \mathrm{O}_{2}$-producing Lactobacillus species in the female genitourinary tract and the use of $\mathrm{H}_{2} \mathrm{O}_{2}$ by the host immune system (Carreras et al., 1994a). Of all the mutants tested, the estD::kan mutant displayed the most unique phenotype. This mutant is not defective in its ability to form biofilm over glass, yet it is severely attenuated in its ability to form biofilm over THCEC. Although aniA::kan and $c c p:: k a n$ are more severely attenuated over THCEC, both mutants have reduced average thicknesses compared to the wild type when cultured over glass. Cervical epithelial and endothelial cells produce NO (Carreras et al., 1994a; Tschugguel et al., 1999; Ledingham et al., 2000), which is reduced to $s$-nitrosoglutathione (GSNO) in the gonococcus (Seib et al., 2006). $\mathrm{NO}$ and subsequently GSNO would likely be more abundant in the THCEC culture system. GSNO is toxic and EstD may have a role in its metabolism in the cell (Seib et al., 2006). This may explain why an estD::kan mutant is severely attenuated over cells, but is not

\section{REFERENCES}

An, D., and Parsek, M. R. (2007). The promise and peril of transcriptional profiling in biofilm communities. Curr. Opin. Microbiol. 10, 292-296.

Anonymous. (2008). Centers for Disease Control and Prevention, Sexually Transmitted Disease Surveillance 2007. The Department of Health and Human Services, Atlanta, GA.

Bala,M., andSood,S.(2010).Cephalosporin resistance in Neisseria gonorrhoeae. J. Glob. Infect. Dis. 2, 284-290.

Barraud, N., Hassett, D. J., Hwang, S. H., Rice, S. A., Kjelleberg, S., and Webb, J. S. (2006). Involvement of nitric oxide in biofilm dispersal of Pseudomonas aeruginosa. J. Bacteriol. 188, 7344-7353.

attenuated over glass. In our glass flow cell system, the most abundant source of $\mathrm{NO}$ would be anaerobic respiration in the gonococcus. However, NO is rapidly reduced by NorB in vitro (Cardinale and Clark, 2005). In contrast to the estD::kan mutant, a trxB::kan mutant is attenuated for biofilm formation over glass and THCEC. TrxB is also involved in NO tolerance, but NO is likely not abundant in our continuous-flow system over glass, as previously discussed. However, the $\operatorname{tr} x B:$ :kan mutant is likely attenuated in both systems, because it is impaired in its ability to undergo anaerobic respiration. We have determined that transcription of both aniA and nor $B$ is reduced in this mutant compared to the wild type. Thus, deficient biofilm formation is likely attributable to the reduced expression of aniA and norB. The phenotype may be more severe over cells where the NO concentration is higher, as the $\operatorname{tr} x B:$ :kan mutant would also lack the ability to efficiently reduce NO. Overall, our findings clearly demonstrate that the ability to tolerate oxidative stress is necessary for robust levels of biofilm formation.

Biofilm formation by N. gonorrhoeae may aid in oxidative tolerance during the cervical infection of women by positively regulating factors, such as $c c p$ and norB, which reduce reactive oxygen and nitrogen species, respectively. Anaerobic respiration in biofilm may also represent an adaptation to oxygen limitation within the biofilm or the host environment. However, biofilm formation may play a more prominent role in oxidative stress defense, as we determined that the majority of the genes involved in these pathways are required for robust biofilm formation. The propensity for biofilms to form during natural cervical infection, the conditions present during male infection that are likely not conducive to biofilm formation, and the lack of evidence of biofilm formation in men, suggests that biofilm formation may be specific to the infection of women. Biofilm formation may confer properties to the gonococcus through the induction of the anaerobic metabolism and oxidative stresses defense pathways, enhancing the ability to cope with or evade the host immune response. The ability to do so likely contributes to the occurrence of persistent infection in women and may help to account for the greater likelihood of asymptomatic infection in women. Mechanisms that govern biofilm formation might be manipulated to improve treatment or diagnosis of N. gonorrhoeae infection in women.

(1994b). Kinetics of nitric oxide and hydrogen peroxide production and formation of peroxynitrite during the respiratory burst of human neutrophils. FEBS Lett. 341, 65-68.

Ceri, H., Olson, M. E., Stremick, C., Read, R. R., Morck, D., and Buret, A. (1999). The Calgary biofilm device: new technology for rapid determination of antibiotic susceptibilities of bacterial biofilms. J. Clin. Microbiol. 37, 1771-1776.

Costerton, J. W., Stewart, P. S., and Greenberg, E. P. (1999). Bacterial biofilms: a common cause of persistent infections. Science 284, 1318-1322.

Davies, D. G., Parsek, M. R., Pearson, J. P., Iglewski, B. H., Costerton, J. W., and Greenberg, E. P. (1998). The involvement of cell-to-cell signals in the development of a bacterial biofilm. Science 280, 295-298.

Davis, K. L., Martin, E., Turko, I. V., and Murad, F. (2001). Novel effects of nitric oxide. Annu. Rev. Pharmacol. Toxicol. 41, 203-236.

Deeudom, M., Rock, J., and Moir, J. (2006). Organization of the respiratory chain of Neisseria meningitidis. Biochem. Soc. Trans. 34, 139-142.

Dorward, D. W., Garon, C. F., and Judd, R. C. (1989). Export and intercellular transfer of DNA via membrane blebs of Neisseria gonorrhoeae. J. Bacteriol. 171, 2499-2505.

Dunne, W. M. Jr. (2002). Bacterial adhesion: seen any good biofilms lately? Clin. Microbiol. Rev. 15, 155-166. 
Edwards, J. L., and Apicella, M. A. (2004). The molecular mechanisms used by Neisseria gonorrhoeae to initiate infection differ between men and women. Clin. Microbiol. Rev. 17, 965-981. [Table of contents].

Falsetta, M. L., Bair, T. B., Ku, S. C., Vanden Hoven, R. N., Steichen, C. T., McEwan, A. G., Jennings, M. P., and Apicella, M. A. (2009). Transcriptional profiling identifies the metabolic phenotype of gonococcal biofilms. Infect. Immun. 77, 3522-3532.

Falsetta, M. L., McEwan, A. G., Jennings, M. P., and Apicella, M. A. (2010). Anaerobic metabolism occurs in the substratum of gonococcal biofilms and may be sustained in part by nitric oxide. Infect. Immun. 78, 2320-2328.

Fang, F.C. (1997). Perspectives series: host/ pathogen interactions. Mechanisms of nitric oxide-related antimicrobial activity. J. Clin. Invest. 99, 2818-2825.

Filiatrault, M. J., Picardo, K. F., Ngai, H., Passador, L., and Iglewski, B. H. (2006). Identification of Pseudomonas aeruginosa genes involved in virulence and anaerobic growth. Infect. Immun. 74, 4237-4245.

Gerbase, A. C., Rowley, J. T., Heymann, D. H., Berkley, S. F., and Piot, P. (1998). Global prevalence and incidence estimates of selected curable STDs. Sex. Transm. Infect. 74(Suppl. 1) S12-S16.

Golparian, D., Hellmark, B., Fredlund, H., and Unemo, M. (2010). Emergence, spread and characteristics of Neisseria gonorrhoeae isolates with in vitro decreased susceptibility and resistance to extended-spectrum cephalosporins in Sweden. Sex. Transm. Infect. 86, 454-460.

Greiner, L. L., Edwards, J. L., Shao, J., Rabinak, C., Entz, D., and Apicella, M. A. (2005). Biofilm Formation by Neisseria gonorrhoeae. Infect. Immun. 73, 1964-1970.

Hamilton, H. L., Dominguez, N. M., Schwartz, K. J., Hackett, K. T., and Dillard, J. P. (2005). Neisseria gonorrhoeae secretes chromosomal DNA via a novel type IV secretion system. Mol. Microbiol. 55, 1704-1721.

Handsfield, H. H., and Sparling, P. F. (2005). "Neisseria gonorrhoeae," in Principles and Practices of Infectious Diseases, ed. G. L. Mandell (New York, NY: Churchill Livingstone, Inc.), 2514-2529.

Hassett, D. J., Cuppoletti, J., Trapnell, B., Lymar, S. V., Rowe, J. J., Yoon, S. S., Hilliard, G. M., Parvatiyar, K., Kamani, M. C., Wozniak, D. J., Hwang, S. H., McDermott, T. R., and Ochsner, U. A. (2002). Anaerobic metabolism and quorum sensing by Pseudomonas aeruginosa biofilms in chronically infected cystic fibrosis airways: rethinking antibiotic treatment strategies and drug targets. Adv. Drug Deliv. Rev. 54, 1425-1443.

Holmes, K. K., Sparling, P. F., Per-Anders, M., Lemon, S. M., Stamm, W. E., Piot, P., and Wasserheit, J. N. (1999). Sexually Transmitted Diseases. New York: McGraw-Hill.

Hopper, A., Tovell, N., and Cole, J. (2009). A physiologically significant role in nitrite reduction of the CcoP subunit of the cytochrome oxidase cbb3 from Neisseria gonorrhoeae. FEMS Microbiol. Lett. 301, 232-240.

Householder, T.C., Belli, W.A., Lissenden, S., Cole, J. A., and Clark, V. L. (1999). cis- and trans-acting elements involved in regulation of aniA, the gene encoding the major anaerobically induced outer membrane protein in Neisseria gonorrhoeae. J. Bacteriol. 181, 541-551.

Householder, T. C., Fozo, E. M., Cardinale, J. A., and Clark, V. L. (2000). Gonococcal nitric oxide reductase is encoded by a single gene, norB, which is required for anaerobic growth and is induced by nitric oxide. Infect. Immun. 68, 5241-5246.

James-Holmquest, A. N., Wende, R. D., Mudd, R. L., and Williams, R. P. (1973). Comparison of atmospheric conditions for culture of clinical specimens of Neisseria gonorrhoeae. Appl. Microbiol. 26, 466-469.

Jennings, J. M., Taylor, R., Iannacchione, V. G., Rogers, S. M., Chung, S. E., Huettner, S., and Ellen, J. M. (2010). The available pool of sex partners and risk for a current bacterial sexually transmitted infection. Ann. Epidemiol. 20, 532-538.

Knapp, J. S., and Clark, V. L. (1984). Anaerobic growth of Neisseria gonorrhoeae coupled to nitrite reduction. Infect. Immun. 46, 176-181.

Ledingham, M.A., Thomson, A. J., Young, A., Macara, L. M., Greer, I. A., and Norman, J. E. (2000). Changes in the expression of nitric oxide synthase in the human uterine cervix during pregnancy and parturition. Mol. Hum. Reprod. 6, 1041-1048.

Lim, K. H., Jones, C. E., vanden Hoven, R. N., Edwards, J. L., Falsetta, M. L., Apicella, M. A., Jennings, M. P., and McEwan, A. G. (2008). Metal binding specificity of the MntABC permease of Neisseria gonorrhoeae and its influence on bacterial growth and interaction with cervical epithelial cells. Infect. Immun. 76, 3569-3576.

MacMicking, J., Xie, Q. W., and Nathan, C. (1997). Nitric oxide and macrophage function. Annu. Rev. Immunol. 15, 323-350.

Mellies, J., Jose, J., and Meyer, T. F. (1997). The Neisseria gonorrhoeae gene aniA encodes an inducible nitrite reductase. Mol. Gen. Genet. 256, 525-532.

Pitcher, R. S., and Watmough, N. J. (2004). The bacterial cytochrome cbb3 oxidases. Biochim. Biophys. Acta 1655, 388-399.

Potter, A. J., Kidd, S. P., Edwards, J. L., Falsetta, M. L., Apicella, M. A., Jennings, M. P., and McEwan, A. G. (2009a). Esterase D is essential for protection of Neisseria gonorrhoeae against nitrosative stress and for bacterial growth during interaction with cervical epithelial cells. J. Infect. Dis. 200, 273-278.

Potter, A. J., Kidd, S. P., Edwards, J. L., Falsetta, M. L., Apicella, M. A., Jennings, M. P., and McEwan, A. G. (2009b). Thioredoxin reductase is essential for protection of Neisseria gonorrhoeae against killing by nitric oxide and for bacterial growth during interaction with cervical epithelial cells. J. Infect. Dis. 199, 227-235.

Rani, S. A., Pitts, B., Beyenal, H., Veluchamy, R. A., Lewandowski, Z., Davison, W. M., Buckingham-Meyer, K., and Stewart, P. S. (2007). Spatial patterns of DNA replication, protein synthesis, and oxygen concentration within bacterial biofilms reveal diverse physiological states. J. Bacteriol. 189, 4223-4233.

Rock, J. D., Mahnane, M. R., Anjum, M. F., Shaw, J. G., Read, R. C., and Moir, J. W. (2005). The pathogen Neisseria meningitidis requires oxygen, but supplements growth by denitrification. Nitrite, nitric oxide and oxygen control respiratory flux at genetic and metabolic levels. Mol. Microbiol. 58, 800-809.

Rock, J. D., Thomson, M. J., Read, R. C., and Moir, J. W. (2007). Regulation of denitrification genes in Neisseria meningitidis by nitric oxide and the repressor NsrR. J. Bacteriol. 189, 1138-1144.

Sauer, K., Camper, A. K., Ehrlich, G. D., Costerton, J. W., and Davies, D. G. (2002). Pseudomonas aeruginosa displays multiple phenotypes during development as a biofilm. J. Bacteriol. 184, 1140-1154.

Schierholz, J. M., Beuth, J., Konig, D., Nurnberger, A., and Pulverer, G. (1999). Antimicrobial substances and effects on sessile bacteria. Zentralbl. Bakteriol. 289, 165-177.

Seib, K. L., Simons, M. P., Wu, H. J., McEwan, A. G., Nauseef, W. M., Apicella, M. A., and Jennings, M. P. (2005). Investigation of oxidative stress defenses of Neisseria gonorrhoeae by using a human polymorphonuclear leukocyte survival assay. Infect. Immun. 73, 5269-5272.

Seib, K. L., Tseng, H. J., McEwan, A. G., Apicella, M. A., and Jennings, M. P.
(2004). Defenses against oxidative stress in Neisseria gonorrhoeae and Neisseria meningitidis: distinctive systems for different lifestyles. J. Infect. Dis. 190, 136-147.

Seib, K. L., Wu, H. J., Kidd, S. P., Apicella, M. A., Jennings, M. P., and McEwan, A. G. (2006). Defenses against oxidative stress in Neisseria gonorrhoeae: a system tailored for a challenging environment. Microbiol. Mol. Biol. Rev. 70, 344-361.

Seib, K. L., Wu, H. J., Srikhanta, Y. N., Edwards, J. L., Falsetta, M. L., Hamilton, A. J., Maguire, T. L., Grimmond, S. M., Apicella, M. A., McEwan, A. G., and Jennings, M. P. (2007). Characterization of the OxyR regulon of Neisseria gonorrhoeae. Mol. Microbiol. 63, 54-68.

Smith, L. D. (1975). The Pathogenic Anaerobic Bacteria. Springfield, IL: Charles C. Thomas Publications, Ltd.

Srikhanta, Y. N., Dowideit, S. J., Edwards, J. L., Falsetta, M. L., Wu, H. J., Harrison, O. B., Fox, K. L., Seib, K. L., Maguire, T. L., Wang, A. H., Maiden, M. C., Grimmond, S.M., Apicella, M.A., and Jennings, M. P. (2009). Phasevarions mediate random switching of gene expression in pathogenic Neisseria. PLoS Pathog. 5, e1000400. doi: 10.1371/journal.ppat. 1000400

Steichen, C., T, Cho, C., Shao, J., and Apicella, M. A. (2011). The Neisseria gonorrhoeae biofilm matrix contains DNA and an endogenous nuclease controls its incorporation. Infect. Immun. 79, 1504-1511.

Steichen, C. T., Shao, J. Q., Ketterer, M. R., and Apicella, M.A. (2008). Gonococcal cervicitis: a role for biofilm in pathogenesis. J. Infect. Dis. 198, 1856-1861.

Tart, A. H., and Wozniak, D. J. (2008). Shifting paradigms in Pseudomonas aeruginosabiofilm research. Curr. Top. Microbiol. Immunol. 322, 193-206.

Tschugguel, W., Schneeberger, C., Lass, H., Stonek, F., Zaghlula, M. B., Czerwenka, K., Schatten, C., Kaider, A., Husslein, P., and Huber, J. C. (1999). Human cervical ripening is associated with an increase in cervical inducible nitric oxide synthase expression. Biol. Reprod. 60, 1367-1372.

Turner, S., Reid, E., Smith, H., and Cole, J. (2003). A novel cytochrome c peroxidase from Neisseria gonorrhoeae: a lipoprotein from a Gram-negative bacterium. Biochem. J. 373, 865-873.

Van Alst, N. E., Picardo, K. F., Iglewski, B. H., and Haidaris, C. G. (2007). Nitrate sensing and metabolism modulate motility, biofilm formation, and virulence in Pseudomonas aeruginosa. Infect. Immun. 75, 3780-3790.

Werner, E., Roe, F., Bugnicourt, A., Franklin, M. J., Heydorn, A., Molin, 
S., Pitts, B., and Stewart, P. S. (2004). Stratified growth in Pseudomonas aeruginosa biofilms. Appl. Environ. Microbiol. 70, 6188-6196.

Whitehead, R. N., Overton, T. W., Snyder, L.A., McGowan, S. J., Smith, H., Cole, J. A., and Saunders, N. J. (2007). The small FNR regulon of Neisseria gonorrhoeae: comparison with the larger Escherichia coli FNR regulon and interaction with theNarQ-NarP regulon. BMCGenomics 8, 35. doi: 10.1186/1471-2164-8-35

Whiteley, M., Lee, K. M., and Greenberg, E. P. (1999). Identification of genes controlled by quorum sensing in Pseudomonas aeruginosa. Proc. Natl. Acad. Sci. U.S.A. 96, 13904-13909.

Wu, H. J., Seib, K. L., Edwards, J. L., Apicella, M. A., McEwan, A. G., and Jennings, M. P. (2005). Azurin of pathogenic Neisseria spp. is involved in defense against hydrogen peroxide and survival within cervical epithelial cells. Infect. Immun. 73, 8444-8448.

Wu, H. J., Seib, K. L., Srikhanta, Y. N., Edwards, J., Kidd, S. P., Maguire, T. L., Hamilton, A., Pan, K. T., Hsiao, H. H., Yao, C. W., Grimmond, S. M., Apicella, M. A., McEwan, A. G., Wang, A. H., and Jennings, M.P.(2010). Manganese regulation of virulence factors and oxidative stress resistance in Neisseria gonorrhoeae. J. Proteomics 73, 899-916.

Wu, H. J., Seib, K. L., Srikhanta, Y. N., Kidd, S. P., Edwards, J. L., Maguire, T. L., Grimmond, S. M., Apicella, M. A., McEwan, A. G., and Jennings, M. P. (2006). PerR controls Mn-dependent resistance to oxidative stress in Neisseria gonorrhoeae. Mol. Microbiol. 60, 401-416

Yoon, S. S., Hennigan, R. F., Hilliard, G. M., Ochsner, U. A., Parvatiyar, K., Kamani, M. C., Allen, H. L., DeKievit,
T. R., Gardner, P. R., Schwab, U., Rowe, J. J., Iglewski, B. H., McDermott, T. R., Mason, R. P., Wozniak, D. J., Hancock, R. E., Parsek, M. R., Noah, T. L., Boucher, R. C., and Hassett, D. J. (2002). Pseudomonas aeruginosa anaerobic respiration in biofilms: relationships to cystic fibrosis pathogenesis. Dev. Cell 3, 593-603.

Zumft, W. G. (1997). Cell biology and molecular basis of denitrification. Microbiol. Mol. Biol. Rev. 61, 533-616.

Conflict of Interest Statement: The authors declare that the research was conducted in the absence of any commercial or financial relationships that could be construed as a potential conflict of interest.

Received: 14 January 2011; paper pending published: 16 February 2011; accepted: 30
March 2011; published online: 18 April 2011.

Citation: Falsetta ML, Steichen CT, McEwan AG, Cho C, Ketterer M, Shao J, Hunt J, Jennings MP and Apicella MA (2011) The composition and metabolic phenotype of Neisseria gonorrhoeae biofilms. Front. Microbio. 2:75. doi: 10.3389/ fmicb.2011.00075

This article was submitted to Frontiers in Cellular and Infection Microbiology, a specialty of Frontiers in Microbiology. Copyright (c) 2011 Falsetta, Steichen, McEwan, Cho, Ketterer, Shao, Hunt, Jennings and Apicella. This is an openaccess article subject to a non-exclusive license between the authors and Frontiers Media SA, which permits use, distribution and reproduction in other forums, provided the original authors and source are credited and other Frontiers conditions are complied with. 